Gut and Liver, Vol. 10, No. 4, July 2016, pp. 489-490

\title{
Is Elderly Age a Simple Predictive Factor for Inadequate Bowel Preparation before Colonoscopy?
}

\author{
Hyun Gun Kim \\ Department of Internal Medicine, Soonchunhyang University College of Medicine, Seoul, Korea
}

See "Effect of Functional Status on the Quality of Bowel Preparation in Elderly Patients Undergoing Screening and Surveillance Colonoscopy" by Akash Kumar, et al. on page 569, Vol. 10. No. 4, 2016

Bowel preparation is important when visualizing the whole colonic mucosa and greatly influences the rate of adenoma detection, which is considered to be a standard indicator of successful colonoscopy. ${ }^{1}$ The target level of adequate preparation (so that repeat colonoscopy is not required within $\leq 1$ year) is $85 \%$ as recommended by the American Society for Gastrointestinal Endoscopy (ASGE)/the American College of Gastroenterology task force. ${ }^{1}$ In recent studies, the bowel preparation rates have reached this target, and low-volume products that effectively cleanse the colon are now available. ${ }^{2}$

However, the bowel preparation rate often fails to meet the expected level in real clinical practice. According to the Clinical Outcomes Research Initiative's national endoscopic database in the United States, preparation was adequate in only $76.9 \%$ of patients. ${ }^{3}$ Inadequate bowel preparation is associated with many negative factors in terms of colorectal cancer screening, such as incomplete colonoscopy, a lower adenoma detection rate, an increased procedure time, an increased chance of adverse events, and increased medical costs because short-term repeat colonoscopy is needed. ${ }^{4}$

Factors contributing to inadequate bowel preparation have been identified in a number of previous studies. ${ }^{5}$ Well-known factors are medical conditions that affect bowel motility including chronic constipation, liver cirrhosis, diabetes, previous intraabdominal surgery, use of multiple medications, use of opioids or tricyclic antidepressants, and conditions associated with immobilization such as older age, poor physical status, obesity, neurological disease, and hospitalization. ${ }^{5}$

In particular, age has been shown to be an independent risk factor for poor bowel preparation regardless of the type of preparation..$^{5-7}$ Recently, the number of colonoscopies performed in elderly and very elderly patients has increased, caused by the global increase in life expectancy. Colonoscopies in elderly examinees are frequently incomplete and carry greater risk of procedural complications. In a recent meta-analysis of colonoscopies in elderly patients, inadequate bowel preparation was documented in $18.8 \%$ of patients $>65$ years of age, and in $12.1 \%$ of those $>80$ years. ${ }^{8}$ However, some studies have shown different results. In prospective studies comparing the success rates and yields of colonoscopy in the elderly with those in younger patients, there were no significant differences in rates of poor bowel preparation (23.4\% vs $19.7 \%$ and $14.3 \%$ vs $20.6 \%)^{9,10}$

Possible explanations for poor bowel preparation in the elderly include delayed gastrointestinal motility, a high rate of constipation, poor compliance, poor understanding of preparation instructions, a high number of previous operations, a high frequency of comorbid diseases (such as diabetes), and other independent factors influencing poor bowel preparation. ${ }^{5,6}$ These problems may be more common as age advances. Thus, recent published studies on colonoscopy in older groups more often investigate octogenarians and nonagenarians than the simply elderly. ${ }^{6,7}$ However, simply comparing elderly or very elderly groups may not be appropriate to obtain a full understanding of poor bowel preparation in older patients. This is because medical conditions that affect bowel motility can differ with individual physical status even in elderly groups and a more detailed approach is needed to understand how best to prepare the bowel in such groups.

On this topic, the study of Kumar et al. ${ }^{11}$ describes an interesting approach and suggests a fresh perspective to bowel preparation for the elderly. In this study, the authors prospectively examined how the functional status of elderly patients

Correspondence to: Hyun Gun Kim

Department of Internal Medicine, Soonchunhyang University College of Medicine, 59 Daesagwan-ro, Yongsan-gu, Seoul 04401, Korea

Tel: +82-2-710-3072, Fax: +82-2-709-9696, E-mail: medgun@schmc.ac.kr

pISSN 1976-2283 eISSN 2005-1212 http://dx.doi.org/10.5009/gnl16203

@) This is an Open Access article distributed under the terms of the Creative Commons Attribution Non-Commercial License (http://creativecommons.org/licenses/by-nc/4.0) which permits unrestricted non-commercial use, distribution, and reproduction in any medium, provided the original work is properly cited. 
affected bowel preparation. Two items including "difficulty with performing activities of daily living" and "difficulty with ambulation" were used to assess functional status. In multivariate analysis, difficulty walking (odds ratio, 5.78; 95\% confidence interval,1.78 to 18.80 ) was shown to be an independent predictor of inadequate preparation. ${ }^{11}$

It is difficult to precisely explain why difficulty walking is associated with nearly 6-fold higher odds of suboptimal preparation. The authors suggested that a possible reason was that this difficulty can interfere with ingestion of the required preparation volume because it requires repeatedly walking to obtain more solution or to the restroom to defecate.

The cited study has important implications. Although difficulty walking may not be uniquely confined to elderly people, the authors indicated that this general characteristic of the elderly may be an important reason for poor bowel preparation. According to the study, ambulation difficulty could significantly predict inadequate bowel preparation in elderly people, apart from advanced age, medical condition, a comorbid disease, or medication use.

Despite the positive implications raised, the cited study has several limitations that prevent generalization of the results to clinical practice. First, the sample size of the study was small. Only 88 elderly people were enrolled in the study and the proportions of patients with strokes or on tricyclic antidepressants were only 6.8\% (each). Also, the age distribution (the proportions of elderly and very elderly people) was not given. The mean age of enrolled patients was 71.6 years, and it thus seems that more elderly than very elderly people participated. Nevertheless, the proportion of patients having difficulty walking was over $40 \%$, and this large proportion of patients with a specific problem compared to the small total sample size might have introduced selection bias considering that the proportions of patients with other clinical characteristics were small. Second, bowel preparation was estimated simply by using a standard institutional four-point scale (following the ASGE recommendations) even though the study was prospective in design. ${ }^{1}$ Also, the rate of adequate bowel preparation was only $70.5 \%$ even though most patients were simply elderly; this rate was considerably lower than that of a prior meta-analysis. ${ }^{8}$ Although the cited study did not compare bowel preparation agents, the acceptable bowel preparation rate might have differed if other validated preparation scores, such as the Boston Bowel Preparation Scale or the Ottawa Bowel Preparation Scale, had been used. ${ }^{12,13}$ Other quality indicators, including the adenoma detection rate, could have been compared between patients who underwent adequate and inadequate bowel preparation to explore the quality of colonoscopy.

The above study, unlike previous studies, shows that there is no need to be apprehensive when elderly examinees must undergo bowel preparation. All possible independent factors that affect bowel preparation quality should be defined, which may include medical conditions, comorbid disease, physical status, and ambulation ability before colonoscopy. Each patient should undergo careful physical examination and give a complete medical history. Simply being elderly does not negatively affect adequate bowel preparation, but an individualized approach is needed for optimal results.

\section{CONFLICTS OF INTEREST}

No potential conflict of interest relevant to this article was reported.

\section{REFERENCES}

1. Rex DK, Schoenfeld PS, Cohen J, et al. Quality indicators for colonoscopy. Gastrointest Endosc 2015;81:31-53.

2. Kim HG, Huh KC, Koo HS, et al. Sodium picosulfate with magnesium citrate (SPMC) plus laxative is a good alternative to conventional large volume polyethylene glycol in bowel preparation: a multicenter randomized single-blinded trial. Gut Liver 2015;9:494-501.

3. Harewood GC, Sharma VK, de Garmo P. Impact of colonoscopy preparation quality on detection of suspected colonic neoplasia. Gastrointest Endosc 2003;58:76-79.

4. Menees SB, Elliott E, Govani S, et al. The impact of bowel cleansing on follow-up recommendations in average-risk patients with a normal colonoscopy. Am J Gastroenterol 2014;109:148-154.

5. Chung YW, Han DS, Park KH, et al. Patient factors predictive of inadequate bowel preparation using polyethylene glycol: a prospective study in Korea. J Clin Gastroenterol 2009;43:448-452.

6. Cha JM. Would you recommend screening colonoscopy for the very elderly? Intest Res 2014;12:275-280.

7. Cha JM, Kozarek RA, La Selva D, et al. Risks and benefits of colonoscopy in patients 90 years or older, compared with younger patients. Clin Gastroenterol Hepatol 2016;14:80-86.e1.

8. Day LW, Kwon A, Inadomi JM, Walter LC, Somsouk M. Adverse events in older patients undergoing colonoscopy: a systematic review and meta-analysis. Gastrointest Endosc 2011;74:885-896.

9. Ma WT, Mahadeva S, Kunanayagam S, Poi PJ, Goh KL. Colonoscopy in elderly Asians: a prospective evaluation in routine clinical practice. J Digestive Dis 2007;8:77-81.

10. Karajeh MA, Sanders DS, Hurlstone DP. Colonoscopy in elderly people is a safe procedure with a high diagnostic yield: a prospective comparative study of 2000 patients. Endoscopy 2006;38:226-230.

11. Kumar A, Lin L, Bernheim O, et al. Effect of functional status on the quality of bowel preparation in elderly patients undergoing screening and surveillance colonoscopy. Gut Liver 2016;10:569-573.

12. Calderwood AH, Jacobson BC. Comprehensive validation of the Boston Bowel Preparation Scale. Gastrointest Endosc 2010;72:686692.

13. Rostom A, Jolicoeur E. Validation of a new scale for the assessment of bowel preparation quality. Gastrointest Endosc 2004;59:482-486. 